\title{
Caracterización Psicológica de un Grupo de Delincuentes Sexuales Chilenos a través del Test de Rorschach
}

\section{Psychological Profile of Chilean Sexual Offenders through the Rorschach Inkblot Test}

\author{
Pamela Jiménez \\ Universidad de La Frontera
}

\begin{abstract}
Es relevante profundizar en las motivaciones que determinan la conducta criminal e intentar una aproximación a la personalidad de quienes delinquen. Así, este estudio tuvo como objetivo determinar la posible existencia de características psicológicas comunes en un grupo de delincuentes sexuales. Con este propósito se utilizó el Test de Rorschach y, como criterio de comparación, datos de individuos con comisión de un delito de hurto y/o robo y datos estadísticos del Rorschach de referencia nacional. Se encontraron diferencias significativas con ambos grupos, lo que sugiere la existencia de un conjunto de características psicológicas en los delincuentes sexuales que les serían propias y les distinguirían tanto del resto de la población como del resto de los delincuentes comunes.
\end{abstract}

Palabras clave: delincuente sexual, evaluación psicológica, Test de Rorschach.

\begin{abstract}
It is relevant to deepen our understanding of the motivations of criminal behaviour and to try to understand such personalities. The objective of this study was to determine if there were underlying psychological characteristics common to sexual offenders. For this purpose, the Rorschach Inkblot test was used and, as comparative parameters, individuals with histories of theft and Chilean national Rorschach statistics. Significant differences were found in both groups, which suggest the existence of underlying common psychological traits in sexual offenders. These traits make them stand out from the general population and from other common delinquents.
\end{abstract}

Keywords: sexual offender, psychological assessment, Rorschach Inkblot Test.

\section{Introducción}

La delincuencia y la conducta criminal se han constituido en materias que generan interés de un gran número de estudiosos, dada la complejidad de factores o posibles predictores asociados a su génesis. Sus investigaciones convergen en una disciplina común que es la Criminología: ciencia integral que estudia el comportamiento delictivo y la reacción social frente al mismo, y comparte algunos

\footnotetext{
Pamela Jiménez Etcheverría, Universidad de La Frontera, Temuco, Chile.

La correspondencia relativa a este artículo debe ser dirigida a Pamela Jiménez Etcheverría, Departamento de Psicología, Magíster en Psicología Jurídica y Forense, Universidad de La Frontera, Casilla 54-D, Temuco, Chile. E-mail: pjimenez@ufro.cl

Este artículo se basa en la tesis para optar al título de Máster en Evaluación Psicológica Clínica y Forense de la Universidad de Salamanca, España.

La autora agradece la valiosa cooperación del psicólogo Manuel Ortiz en la revisión de los aspectos metodológicos del estudio.
}

conocimientos, términos e instrumentos de investigación con otras disciplinas, como la sociología, la psicología, la medicina, la biología y el derecho (Garrido, Stangeland \& Redondo, 1999).

La psicología jurídica engloba varias especialidades bien diferenciadas; aparece entre ellas y como una subespecialidad claramente distinguible la psicología forense (Sobral, Arce \& Prieto, 1994). De las actuaciones del psicólogo en este ámbito, destaca la práctica pericial orientada a juzgar las alteraciones del comportamiento humano y de las relaciones interpersonales, dentro del marco que propone la ley.

La legislación chilena enfatiza regular el castigo de quienes presentan conductas que se desvíen o transgredan las normas. Cabe preguntarse ¿qué sucede con la rehabilitación? Es cierto que algunos delincuentes son muy peligrosos, pero no es menos cierto que hay quienes podrían disminuir la reincidencia si existieran programas que resultaran efectivos. En relación a la intervención y tratamiento de los delincuentes, Garrido (1993) sostiene que es necesaria, desde el punto de vista del bienestar de los 
internos, de la sociedad y de los propios funcionarios penitenciarios.

Reconociendo la importancia de abordar el fenómeno delictual desde un enfoque que integre tanto el trasfondo social como la psicología de quienes delinquen, para comprender el fenómeno en su totalidad aparece relevante considerar las variables individuales, pues, si bien el individuo es un producto de la influencia ambiental, no deja por eso de ser alguien diferente del resto, con motivaciones propias y peculiares.

Por ello, para avanzar en la disminución de la delincuencia es necesario conocer las motivaciones o el funcionamiento particular del individuo que comete este tipo de actos para poder así intervenir en el diagnóstico y prevención de este fenómeno. Así, desde la psicología, tal como señala Romero (1999, citado en Sierra, Jiménez \& Buela-Casal, 2006), la mayor parte de las explicaciones de la conducta criminal se ocupan de la criminalidad o tendencia a implicarse en actos delictivos.

Romero (1999, citado en Sierra et al., 2006) plantea que las principales corrientes de estudio sobre factores psicológicos explicativos de la conducta criminal se dividen en aquellas que destacan los factores de carácter psicosocial (influencia de los contextos de socialización) y aquellas que destacan las características más individuales. En esta segunda línea, las variables de personalidad más estudiadas como posibles predictoras de la conducta se dividen en una perspectiva temperamental y una corriente socio-cognitiva. En la primera se propone, entre otros aspectos, que las dificultades para reflexionar antes de actuar o para considerar las consecuencias negativas de la conducta ponen al individuo en riesgo de implicación delictiva. En el polo socio-cognitivo se han encontrado resultados contradictorios respecto de la relación entre autoestima y conducta antisocial, así como datos poco consistentes para explicar la relación que presenta con las habilidades sociales. Sin embargo, sí existe consenso para señalar que son múltiples los factores implicados en la conducta criminal y en la necesidad de estudiar los efectos moduladores (de interacción) entre diferentes factores (para una exposición completa y detallada del tema véase Romero \& Luengo, 1999).

Dentro de la delincuencia violenta, destaca la de carácter sexual, por suponer un fuerte atropello a la libertad de las personas. Las agresiones sexuales constituyen un determinado tipo de parafilias -conductas sexuales poco comunes o extrañas- que tienen la peculiaridad de suponer una transgresión del libre consentimiento de otra persona (Echeburúa, 1994). Según Barudy (1999), estos delitos constituyen un profundo y grave atentado a la integridad física o psicológica de las víctimas, comparable a una "tentativa de asesinato moral".

En la literatura el delito sexual es asociado a características psicológicas, en contraposición a las explicaciones más ambientalistas y sociológicas de la delincuencia común, en el sentido que esta última supone un beneficio económico y material para el delincuente (Garrido, 1993). Para conocer el perfil de los delincuentes sexuales, la investigación psicológica ha permitido obtener información valiosa sobre su personalidad, aumentando la comprensión del fenómeno de la delincuencia sexual y las posibilidades de intervención sobre el mismo.

A partir del estudio de la delincuencia sexual, autores como Echeburúa y Guerricaechevarría (2000), Barudy (1999) y Garrido (1993) describen al delincuente sexual como mayormente de sexo masculino (asociado al mayor impulso sexual y componente agresivo presente en los varones), con trastornos de la identidad, la autoestima y las capacidades para establecer y mantener relaciones interpersonales. Señalan, además, que este aparece inseguro, inmaduro, poco asertivo y con baja capacidad empática, con baja disposición para ajustarse a las normas y con una marcada tendencia a la distorsión. Diversos autores consideran a esta última como una de las características más importantes en los delincuentes sexuales. Esta característica daría cuenta de un estilo de funcionamiento en que la persona se crea una idea, se forma una opinión sobre lo que desearía que pasara y actúa como si fuera a ocurrir de verdad, sin considerar las probabilidades reales, ignorando las reglas sociales y siguiendo sus propios criterios, lo que potencia la falta de respeto por los derechos y sentimientos de los demás.

Beneyto y Garrido (1997) señalan, además, que la conducta delictiva sexual suele manifestarse junto a dificultades para establecer relaciones íntimas, emocionales y sexuales. Describen al delincuente sexual como una persona que experimenta impotencia y falta de asertividad con las mujeres, junto a un déficit en el control de su impulso sexual.

Basándose en las características observadas en los delincuentes sexuales, varios autores han formulado distintas tipologías que agruparían a los agresores sexuales dependiendo del tipo de agresión 
sexual, de la víctima, de la relación existente con esta y de los mecanismos psicológicos a la base de dicha conducta (Calderón, Duguet, Espinoza \& Fuentealba, 2001).

Gómez (1999) señala que se pueden clasificar los agresores sexuales en cuatro grupos en función del tipo de víctima: (1) agresores sexuales en ámbito familiar, (2) agresores sexuales a desconocidas, (3) agresores sexuales a conocidas y (4) agresores sexuales a menores.

El grupo perteneciente a los agresores sexuales en ámbito familiar utiliza la confianza puesta en ellos para actuar; son agresores posesivos que utilizan el miedo para paralizar a la víctima y manifiestan un bajo nivel de autoestima.

En el grupo de agresión a desconocidas, la agresión tiene un móvil puramente agresivo y no sexual. Las principales características personales del agresor dan cuenta de pobres habilidades sociales, gran inmadurez afectiva y bajo nivel de autoestima.

El tercer grupo, agresores sexuales que han establecido una corta relación previa de tipo amistoso con la víctima, suele tener un desarrollo socializador precario, con escasos recursos personales y bajo nivel de autocontrol.

Especial mención hace Gómez (1999) a las agresiones sexuales a menores, señalando que en estos casos analizar a los agresores es muy difícil ya que estos no aceptan su autoría, dificultando efectuar una historia de la conducta problema; de los limitados datos obtenidos, deduce que el agresor considera que la relación con el menor es solo una demostración de afecto. Al tener graves dificultades de relación afectiva y sexual con los adultos, este agresor recurre a los menores sobre los que puede actuar mediante su superioridad, con lo que muestra una personalidad inmadura, desequilibrio afectivo, déficit en habilidades sociales y dificultades para establecer vínculos afectivos estables.

En todos los casos de agresores sexuales, Gómez (1999) sostiene que es posible realizar tratamiento psicológico para producir una mejoría en su estabilidad psíquica, considerando que si el agresor posee recursos intelectuales y culturales más elevados, es capaz de modificar los criterios alterados a nivel cognitivo, si bien el cambio a nivel emocional y comportamental es más difícil. A medida que desciende el nivel de recursos del agresor existe más resistencia a una modificación, aunque solo sea a nivel cognitivo, de las ideas que rigen su concepto de los roles masculino y femenino.
Debido a su escasez de recursos, el pensamiento de estos individuos es más rígido y estereotipado que lo normal. Su resistencia al cambio se fundamenta en la imposibilidad de hacer un análisis racional de los principios que han motivado la oposición y persistencia de dichas ideas.

\section{Prevalencia de la Delincuencia Sexual}

Según estadísticas entregadas por Gendarmería de Chile (Región de la Araucanía), se encontró que los delitos sexuales corresponden a un $17,3 \%$ del total de los procesados ingresados desde Diciembre de 1999 a Mayo del 2000.

Cifras recogidas en la Región de la Araucanía indican que la violación es más frecuente en niñas menores de 18 años, representando estas a un $45 \%$ de las víctimas. En cuanto a los agresores, el 59\% son adultos, de los cuales la mitad tiene educación básica y solo un $13 \%$ de ellos llega a ser detenido. La sodomía afecta a menores de edad en el $57 \%$ de las denuncias, siendo el autor, en un $42 \%$ de los casos, un adulto; solo un 14\% queda en calidad de detenido. Por su parte, el incesto tiene un 50\% de víctimas mujeres, casi en su mayoría menores (33\%) y su agresor es en un 50\% varón, alcanzando un $33 \%$ la educación básica, no teniéndose registros de detenidos (Hermosilla, 1997).

En Chile, en los registros a Diciembre del 2001 los delincuentes sexuales representaban el 7,5\% de los 20.568 encarcelados (Gendarmería de Chile, 2001). Se señala que los delitos sexuales son los que más cifras negras ocultan. La incertidumbre respecto a la cantidad de casos, se debe, en parte, a que no existe un registro unificado de información (Bravo, 1982). No obstante, en los últimos años, las denuncias por delitos sexuales han ido aumentando (Montoya, Díaz, Reyes, Abusleme \& Garrido, 2004). En el año 2006 hubo 15.209 casos de delitos sexuales denunciados en Chile, de los cuales el 61,4\% tuvo como víctima a un menor de edad. El mayor número de víctimas estuvo en el rango etario de 7 a 14 años (4.446), seguido de los adolescentes de 14 a 18 años (3.757) y de menores de 7 años (2.234). Respecto del género, las estadísticas indican una relación de cuatro mujeres por cada hombre víctima de delitos de este tipo. Además, de acuerdo a las estadísticas de victimización, un $75 \%$ de los agresores corresponde a personas cercanas a la víctima, encabezando el estado de parentesco el padre biológico de la víctima, seguido por el padrastro ( $\mathrm{La}$ Nación, 2007, Agosto 9). 


\section{Antecedentes Empíricos}

Investigaciones realizadas desde el campo de la criminología han utilizado material psicométrico, en un intento por definir y describir una psicología diferencial del transgresor violento (Wolfgang \& Ferracutti, 1971). Bouzat y Pinatel (1975) señalan que la metodología usada en las investigaciones criminológicas -específicamente en el examen psicológico- se apoya en los test, y concretamente en test proyectivos, los cuales revelan las imágenes y los sentimientos que la persona es incapaz o poco dispuesta a manifestar. Así, el individuo analizado se revela, generalmente, sin que él mismo lo sepa.

Wolfgang y Ferracutti (1971) afirman que tanto el Test de Rorschach como el Test de Apercepción Temática permiten diferenciar grupos de extrema agresividad y extrema no agresividad.

A través del Rorschach se manifiesta una conducta agresiva en un bajo porcentaje de respuestas con contenido humano entre los transgresores contra las personas. La frecuencia de contenidos humanos por debajo de la media se dan más entre personas que no parecen identificarse estrechamente con los valores sociales imperantes (Exner, 1994). Si se acompaña de color puro y espacio en blanco (S), indicaría tendencias criminales, carencia de empatía, dificultad en identificarse con los otros y marcado narcisismo (Weigle, 1988).

En base a un estudio realizado en Ecuador con población penal utilizando el Test de Rorschach, Endara (1960) concluye que la carencia de respuestas de contenido humano revela agresividad y falta de empatía. Si bien estas conclusiones fueron derivadas de investigaciones que datan de la década del 60, estudios más recientes las avalan. Así, en Argentina Miotto (1989, Julio), utilizando el Rorschach en un grupo de homicidas, encontró bajo número de respuestas, bajo porcentaje de determinantes múltiples, escaso número de respuestas $\mathrm{M}$, bajo número de respuestas populares, respuestas $\mathrm{FC}$ en bajo número o ausentes y alto número de respuestas de contenido animal.

El espacio en blanco en el Rorschach (S) se ha encontrado en individuos de marcada agresividad. Exner (1994) afirma que si el valor de S es 4 ó más, y al menos una $\mathrm{S}$ aparece después de la lámina III, señala la presencia de una actitud claramente rabiosa y negativa hacia el entorno y constituye un rasgo caracterial; si existen problemas de control y modulación, es probable que las manifestaciones intensas de afectividad incluyan la expresión abierta de esta predisposición oposicionista del individuo.

\section{Objetivos e Hipótesis}

La presente investigación tuvo como objetivo la descripción de las características de personalidad presentes en un grupo de condenados por la comisión de un delito sexual, con el fin de contribuir a la comprensión de esta conducta criminal desde una perspectiva psicológica. En la contrastación de los resultados se utilizaron los datos del estudio de referencia nacional obtenidos por Vinet (2000), pues se acercan más a la población de la cual provienen los reclusos. Se trabajó, también, con los datos obtenidos de un grupo de condenados por el delito de hurto y robo (Espejo, Jiménez \& Mella, 1996), con el fin de determinar qué características psicológicas propias del grupo de delincuentes sexuales permiten distinguirlo del grupo de delincuentes comunes.

Como hipótesis general se propuso que existen características psicológicas compartidas por un grupo de reclusos de sexo masculino condenados por haber cometido un delito sexual, observables a través de indicadores Rorschach, y que se diferencian significativamente de las características psicológicas de los datos del estudio de referencia nacional y de los indicadores Ro del grupo de delincuentes comunes.

Además, se formularon las siguientes hipótesis específicas:

H1: El grupo de individuos condenados por la comisión de un delito sexual presenta indicadores Ro que se diferencian de los datos del estudio de referencia nacional:

H1.1 Presenta indicadores que revelan habilidades sociales y de adaptación al entorno significativamente menores a los del estudio de referencia nacional.

H1.2 Presenta indicadores que revelan distorsión cognitiva significativamente mayor a los del estudio de referencia nacional.

H2: $\quad$ El grupo de individuos condenados por delito sexual presenta indicadores Ro que se diferencian de los datos obtenidos en el grupo de delincuentes comunes:

H2.1 Presenta indicadores que revelan habilidades sociales y de adaptación al entorno significativamente menores a las de los delincuentes comunes.

H2.2 Presenta indicadores que revelan distorsión cognitiva significativamente mayor a los delincuentes comunes. 


\section{Método}

\section{Diseño}

Para la realización de este estudio se utilizó un diseño no experimental, transversal, descriptivo. Para contrastar los resultados de esta investigación, se utilizó como criterio el estudio de referencia nacional de Vinet (2000) y los datos de un grupo de delincuentes comunes (Espejo et al., 1996).

\section{Variables}

La variable independiente fue la comisión de delito, considerándose tres valores: a) delincuente sexual, representado por una muestra de 20 individuos condenados y recluidos por el delito de homicidio; b) no comisión de delito, representada por los datos del estudio de referencia nacional de Vinet (2000); y c) delincuente común, representado por una muestra de 30 personas condenadas y recluidas por los delitos de hurto y robo sin violencia física a terceros.

La variable dependiente fueron los indicadores Rorschach. Operacionalmente se diferenció un número limitado de variables (44 para comparar con el grupo de delincuentes comunes y 51 con el estudio de referencia nacional), de acuerdo a conveniencia, determinada por las variables o indicadores frecuentemente utilizados en investigaciones previas.

\section{Participantes}

Por medio de un muestreo no probabilístico intencionado se seleccionó desde el Centro de Cumplimiento Penitenciario de Temuco, Región de la Araucanía, Chile, a una muestra de 20 reclusos que cumplieron con los siguientes criterios de inclusión: a) sexo masculino, b) mayores de 18 años, c) de procedencia urbana y rural, d) detenidos y procesados por la comisión de un delito sexual en sus diferentes manifestaciones (ver Anexo). La muestra quedó constituida por un grupo de individuos de los cuales el $60 \%$ era soltero, el $25 \%$ era casado y el restante $15 \%$ era separado. Por otra parte, el $75 \%$ cometió un delito sexual hacia un menor y el $25 \%$ lo hizo hacia un adulto. Las personas de la muestra tenían un promedio de 7 años de educación formal y entre un mínimo de 2 y un máximo de 18 años cumplidos de reclusión. El menor de los reclusos tenía 23 años y el mayor 58. La Tabla 1 presenta la composición de la muestra en algunas de las variables antes descritas.
Tabla 1

Frecuencia y Distribución Porcentual en la Muestra de las Variables Edad, Reclusión y Reincidencia

\begin{tabular}{lrr}
\hline \multicolumn{1}{c}{ Variables } & $f$ & $\%$ \\
\hline Edad & & \\
18-30 & 3 & 15 \\
31-45 & 11 & 55 \\
46 ó más & 16 & 30 \\
Educación (en años) & & \\
0-4 & 5 & 25 \\
5-8 & 9 & 45 \\
9-12 & 6 & 30 \\
Reclusión (en años) & & \\
1-5 & 14 & 70 \\
6-11 & 4 & 20 \\
12-18 & 2 & 10 \\
Reincidencia & & \\
Sí & 7 & 35 \\
No & 13 & 65 \\
Total & 20 & 100 \\
\hline
\end{tabular}

\section{Instrumento}

El instrumento utilizado fue el Test de Rorschach, el cual, a través de una serie de estímulos inestructurados, permite obtener información sobre la estructura y dinámica de personalidad de los individuos, lo que resulta útil para conocer las motivaciones de quien comete un delito, las que, incluso, pudiendo ser desconocidas para él, quedan de manifiesto a través de esta prueba. Las respuestas al test fueron codificadas e interpretadas de acuerdo al sistema comprehensivo (Exner, 1994), el cual posee entre sus características distintivas la presentación de datos normativos para un gran número de variables que sirven de referentes grupales para la contrastación de datos individuales para facilitar la interpretación (Vinet, 1995).

\section{Procedimiento}

El procedimiento utilizado para la obtención de los datos de la investigación se inició a partir del contacto oficial con la Dirección del Centro de Cumplimiento Penitenciario (CCP) de Temuco; posteriormente se revisaron los expedientes de los reclusos en este Centro por un delito sexual, luego se realizó la preselección intencionada de la muestra. Se entrevistó a 32 individuos; de estos, 25 accedie- 
ron a participar en el estudio. Se realizó la administración del test de Rorschach a los 25 participantes, siguiendo el procedimiento de administración indicado por el sistema comprehensivo de Exner. De los 25 participantes, 20 contestaron en forma completa, quedando la muestra constituida finalmente por 20 individuos condenados por un delito sexual.

\section{Análisis de Datos}

Los protocolos de respuesta de cada participante fueron codificados utilizando los criterios del sistema comprehensivo de Exner. Luego fueron digitados a través del programa computacional Rorschach Interpretation Assistance Program (RIAP 3.0; Exner \& Tuttle, 1994).

Luego se construyó una base de datos en el procesador estadístico SPSS 16.0. Se optó por 51 indicadores por razones de conveniencia, debido a que, como ya se señaló, estos son comunes a los estudiados en las investigaciones que se emplean como criterio de comparación.

Para analizar H1 y H2 se compararon los promedios y desviaciones estándar de 51 indicadores Rorschach del grupo de individuos que han cometido un delito sexual con los promedios y desviaciones estándar de los datos del estudio de referencia nacional (Vinet, 2000), y los promedios y desviaciones estándar de 44 indicadores Rorschach del grupo de delincuentes sexuales con los datos del grupo de delincuentes comunes (Espejo et al., 1996), por medio de la prueba $t$ de Student.

\section{Resultados}

En relación a $\mathrm{H} 1$, se encontraron diferencias significativas con los datos del estudio de referencia nacional en $16(31 \%)$ de los 51 indicadores (ver Tabla 2): el grupo que ha cometido un delito sexual presenta valores significativamente mayores en un indicador (pasivo) y valores significativamente menores en 15 de ellos ( $\mathrm{R}, \mathrm{DQo}, \mathrm{X}+\%, \mathrm{FC}, \mathrm{CF}$, SumPondC, Lambda, EA, Sum Y, F, Ma, H, (Hd), Hd, PER). Estos resultados sugieren la existencia de un conjunto de características psicológicas en los delincuentes sexuales que le serían propias y los distinguiría del resto de la población: un estilo de manejarse consigo mismos y con su entorno en forma pasiva, inmadura y con un pobre contacto con la realidad.

Específicamente, se observan diferencias significativas en los indicadores más relevantes asociados a las habilidades sociales -SumPond C, EA, H-, los que resultan menores en los delincuentes sexuales que en el estudio de referencia nacional. Esto indica que el monto de recursos con que cuentan no sería suficiente para desarrollar adecuadas habilidades sociales, como tampoco para enfrentar las demandas del medio, siendo así limitada su capacidad para reconocer las necesidades de otros y de adaptación al entorno. Por lo tanto, los resultados apoyan la H1.1.

Del mismo modo, se encontró que uno de los indicadores Rorschach que entrega información sobre ajuste perceptivo $(\mathrm{X}+\%)$ presenta diferencias al comparar el grupo de delincuentes sexuales con el grupo de referencia nacional, siendo significativamente menor en los delincuentes sexuales. Así, los resultados apoyan la H1.2.

En cuanto a la $\mathrm{H} 2$, se encontraron diferencias entre el grupo de delincuentes sexuales y el grupo de delincuentes comunes en 13 (30\%) de los 44 indicadores (ver Tabla 3 ). Se observó que el primer grupo presenta valores significativamente mayores en ocho de los indicadores (W, DQ+, Xu\%, pasivo, $\mathrm{Zf}, \mathrm{Cg}$, Idio, PER) y valores significativamente menores en cinco de ellos (DQo, X-\%, FC, (Hd), Hd). Estos resultados sugieren que las características psicológicas encontradas en el grupo de delincuentes sexuales, no solo los distingue del resto de la población, sino también de los delincuentes comunes. Los datos evidenciarían que los delitos sexuales se caracterizan por mayor impulsividad, subjetividad y autoritarismo, y carencia de enfoque práctico, al compararlos con otras conductas delictivas comunes.

Específicamente, se observan diferencias significativas en indicadores asociados a las habilidades sociales -pasivo, Cg, PER-, los que presentan valores mayores en los delincuentes sexuales que en los delincuentes comunes, como también en indicadores asociados al enfoque de la realidad -W y $\mathrm{Zf}-$, los que también son mayores en el grupo estudiado. Del mismo modo, el grupo de delincuentes sexuales presenta valores significativamente mayores en los indicadores $\mathrm{Xu} \%$ e Idio. Estos datos indican un estilo relacional pasivo, superficial y autoritario, que impide un enfrentamiento adecuado a las demandas del medio y de adaptación al entorno, junto a una percepción de tipo subjetiva y personal, con un enfoque muy abarcativo o globalizador, lo que los puede hacer descuidar detalles importantes. Por lo tanto, aparecen plausibles las H2.1y H2.2. 
Tabla 2

Medias, Desviaciones Estándar y Valores t en los 51 Indicadores Rorschach en el Grupo de Delincuentes Sexuales y el Estudio de Referencia Nacional

\begin{tabular}{|c|c|c|c|c|c|c|}
\hline \multirow[b]{2}{*}{ Indicadores } & \multicolumn{2}{|c|}{ Delincuentes Sexuales } & \multicolumn{2}{|c|}{ Referencia Nacional } & \multirow[b]{2}{*}{$t$} & \multirow[b]{2}{*}{$p$} \\
\hline & $M$ & DS & $M$ & $D S$ & & \\
\hline $\mathrm{R}$ & 16,50 & 3,35 & 20,65 & 6,70 & $-5,47$ & $0,00 *$ \\
\hline W & 7,45 & 3,18 & 7,47 & 3,95 & $-0,02$ & 0,97 \\
\hline $\mathrm{D}$ & 7,20 & 4,75 & 9,07 & 4,72 & $-1,76$ & 0,09 \\
\hline $\mathrm{DQ}^{+}$ & 4,20 & 2,04 & 3,85 & 2,64 & 0,76 & 0,45 \\
\hline DQo & 9,70 & 3,02 & 14,59 & 6,44 & $-7,22$ & $0,00 *$ \\
\hline $\mathrm{X}+\%$ & 0,43 & 0,14 & 0,51 & 0,16 & $-2,37$ & $0,02 *$ \\
\hline $\mathrm{F}+\%$ & 0,40 & 0,20 & 0,49 & 0,23 & $-1,95$ & 0,06 \\
\hline X-\% & 0,17 & 0,10 & 0,17 & 0,12 & 0,10 & 0,91 \\
\hline $\mathrm{Xu} \%$ & 0,36 & 0,14 & 0,31 & 0,12 & 1,53 & 0,14 \\
\hline Aislam/R & 0,25 & 0,18 & 0,23 & 0,19 & 0,43 & 0,05 \\
\hline M & 1,18 & 1,24 & 2,37 & 1,99 & $-2,05$ & 0,66 \\
\hline FM & 3,35 & 2,64 & 2,93 & 2,12 & 0,71 & 0,48 \\
\hline $\mathrm{m}$ & 1,55 & 1,43 & 1,39 & 1,66 & 0,50 & 0,62 \\
\hline $\mathrm{FM}+\mathrm{m}$ & 4,95 & 2,87 & 4,31 & 2,98 & 0,99 & 0,33 \\
\hline $\mathrm{FC}$ & 0,50 & 0,76 & 1,45 & 1,31 & $-5,58$ & $0,00 *$ \\
\hline $\mathrm{CF}$ & 0,55 & 0,60 & 0,85 & 1,17 & $-2,21$ & $0,03 *$ \\
\hline SumPondC & 1,42 & 1,23 & 2,10 & 1,90 & $-2,43$ & $0,02 *$ \\
\hline SumC' & 0,95 & 1,28 & 1,00 & 1,25 & $-0,17$ & 0,86 \\
\hline SumV & 0,25 & 0,64 & 0,33 & 0,64 & $-0,56$ & 0,58 \\
\hline SumT & 0,25 & 0,55 & 0,19 & 0,47 & 0,48 & 0,63 \\
\hline SumY & 0,30 & 0,66 & 1,03 & 1,28 & $-4,96$ & $0,00 *$ \\
\hline $\mathrm{F}$ & 7,55 & 3,47 & 10,26 & 5,57 & $-3,49$ & $0,00 *$ \\
\hline Ego & 0,31 & 0,16 & 0,34 & 0,17 & $-0,83$ & 0,41 \\
\hline Lambda & 1,03 & 0,80 & 1,44 & 1,44 & $-2,26$ & $0,03 *$ \\
\hline EA & 3,37 & 1,93 & 4,48 & 2,96 & $-2,55$ & $0,01 *$ \\
\hline es & 6,65 & 3,21 & 6,87 & 4,04 & $-0,30$ & 0,76 \\
\hline $\mathrm{D}$ & $-0,90$ & 1,20 & $-0,69$ & 1,07 & $-0,77$ & 0,44 \\
\hline Dadj & $-0,65$ & 1,09 & $-0,33$ & 0,84 & $-1,31$ & 0,20 \\
\hline Activo & 2,85 & 2,30 & 3,87 & 3,05 & $-1,98$ & 0,06 \\
\hline Pasivo & 3,95 & 2,25 & 2,88 & 2,26 & 2,11 & $0,04 *$ \\
\hline $\mathrm{Ma}$ & 0,55 & 0,60 & 1,39 & 1,54 & $-6,21$ & $0,00 *$ \\
\hline $\mathrm{Mp}$ & 1,35 & 1,35 & 1,01 & 1,17 & 1,12 & 0,27 \\
\hline $\mathrm{Zf}$ & 8,80 & 3,10 & 9,03 & 4,12 & $-0,33$ & 0,74 \\
\hline $\mathrm{Zd}$ & $-0,45$ & 3,51 & $-1,12$ & 3,92 & 0,85 & 0,40 \\
\hline Afr & 0,46 & 0,16 & 0,49 & 0,16 & $-0,77$ & 0,44 \\
\hline Populares & 3,95 & 1,82 & 4,59 & 1,74 & $-1,57$ & 0,13 \\
\hline $\mathrm{H}$ & 1,55 & 1,05 & 2,23 & 1,70 & $-2,89$ & $0,00 *$ \\
\hline$(\mathrm{H})$ & 1,20 & 1,47 & 0,62 & 0,91 & 1,76 & 0,09 \\
\hline Hd & 0,45 & 0,60 & 1,08 & 1,31 & $-4,65$ & $0,00 *$ \\
\hline (Hd) & 0,05 & 0,22 & 0,31 & 0,66 & $-5,20$ & $0,00 *$ \\
\hline A & 7,90 & 2,97 & 7,49 & 3,27 & 0,61 & 0,54 \\
\hline Art & 0,85 & 1,46 & 0,77 & 1,10 & 0,24 & 0,80 \\
\hline $\mathrm{Cg}$ & 1,25 & 1,33 & 0,68 & 1,26 & 1,91 & 0,07 \\
\hline $\mathrm{Sx}$ & 0,40 & 0,88 & 0,22 & 0,83 & 0,95 & 0,36 \\
\hline Idio & 1,10 & 1,02 & 1,35 & 1,42 & $-1,09$ & 0,28 \\
\hline Sum6CCEE & 0,90 & 1,02 & 1,29 & 1,51 & $-1,70$ & 0,10 \\
\hline SumPond6 & 3,15 & 3,91 & 3,88 & 5,34 & $-0,83$ & 0,41 \\
\hline $\mathrm{AG}$ & 0,15 & 0,37 & 0,25 & 0,49 & $-1,22$ & 0,23 \\
\hline COP & 0,50 & 0,76 & 0,36 & 0,65 & 0,82 & 0,42 \\
\hline MOR & 0,60 & 1,23 & 0,74 & 1,33 & $-0,50$ & 0,61 \\
\hline PER & 0,90 & 0,39 & 0,98 & 1,80 & $-12,78$ & $0,00 *$ \\
\hline
\end{tabular}

\footnotetext{
Notas. Delincuentes sexuales $n=20$; Referencia nacional $n=180$. $M=$ Media.

$D S=$ Desviación estándar.

Se han marcado los $\mathrm{p}<0,05$ con un asterisco.
} 
Tabla 3

Medias, Desviaciones Estándar y Valores t en los 44 Indicadores Rorschach en el Grupo de Delincuentes Sexuales y el Grupo de Delincuentes Comunes

\begin{tabular}{|c|c|c|c|c|c|c|}
\hline \multirow[b]{2}{*}{ Indicadores } & \multicolumn{2}{|c|}{ Delincuentes Sexuales } & \multicolumn{2}{|c|}{ Delincuentes Comunes } & \multirow[b]{2}{*}{$t$} & \multirow[b]{2}{*}{$p$} \\
\hline & $M$ & $D S$ & $M$ & $D S$ & & \\
\hline $\mathrm{R}$ & 16,50 & 3,35 & 17,33 & 3,35 & $-1,04$ & 0,31 \\
\hline W & 7,45 & 3,18 & 5,83 & 2,91 & 2,27 & $0,03 *$ \\
\hline D & 7,20 & 4,75 & 8,66 & 4,08 & $-1,37$ & 0,18 \\
\hline $\mathrm{DQ}^{+}$ & 4,20 & 2,04 & 2,60 & 2,08 & 3,50 & $0,00 *$ \\
\hline DQo & 9,70 & 3,02 & 13,70 & 2,95 & $-5,90$ & $0,00 *$ \\
\hline$X+\%$ & 0,43 & 0,14 & 0,46 & 0,11 & $-0,73$ & 0,47 \\
\hline $\mathrm{F}+\%$ & 0,40 & 0,20 & 0,45 & 0,15 & $-1,06$ & 0,30 \\
\hline $\mathrm{X}-\%$ & 0,17 & 0,10 & 0,23 & 0,12 & $-2,48$ & $0,02 *$ \\
\hline $\mathrm{Xu} \%$ & 0,36 & 0,14 & 0,27 & 0,12 & 2,73 & $0,01 *$ \\
\hline M & 1,18 & 1,24 & 1,97 & 2,10 & $-0,61$ & 0,54 \\
\hline FM & 3,35 & 2,64 & 2,83 & 2,00 & 0,88 & 0,39 \\
\hline $\mathrm{m}$ & 1,55 & 1,43 & 1,16 & 1,31 & 1,21 & 0,23 \\
\hline $\mathrm{FM}+\mathrm{m}$ & 4,95 & 2,87 & 4,00 & 2,87 & 1,47 & 0,15 \\
\hline $\mathrm{FC}$ & 0,50 & 0,76 & 1,10 & 1,26 & 3,52 & $0,00 *$ \\
\hline $\mathrm{CF}$ & 0,55 & 0,60 & 0,30 & 0,53 & 1,84 & 0,08 \\
\hline SumPondC & 1,42 & 1,23 & 1,43 & 1,29 & $-0,01$ & 0,98 \\
\hline $\mathrm{F}$ & 7,55 & 3,47 & 7,80 & 2,94 & $-0,32$ & 0,75 \\
\hline EGO & 0,31 & 0,16 & 0,28 & 0,14 & 0,81 & 0,42 \\
\hline Lambda & 1,03 & 0,80 & 1,04 & 0,61 & $-0,03$ & 0,96 \\
\hline EA & 3,37 & 1,93 & 3,38 & 2,61 & $-0,01$ & 0,99 \\
\hline es & 6,65 & 3,21 & 6,66 & 3,41 & $-0,01$ & 0,98 \\
\hline $\mathrm{D}$ & $-0,90$ & 1,20 & $-0,83$ & 1,28 & $-0,25$ & 0,79 \\
\hline Adj D & $-0,65$ & 1,09 & $-0,56$ & 1,10 & $-0,36$ & 0,71 \\
\hline Activos & 2,85 & 2,30 & 3,23 & 2,54 & $-0,73$ & 0,46 \\
\hline Pasivos & 3,95 & 2,25 & 2,70 & 1,89 & 2,47 & $0,02 *$ \\
\hline $\mathrm{Ma}$ & 0,55 & 0,60 & 0,83 & 1,36 & $-2,07$ & 0,05 \\
\hline $\mathrm{Mp}$ & 1,35 & 1,35 & 1,16 & 1,44 & 0,63 & 0,53 \\
\hline $\mathrm{Zf}$ & 8,80 & 3,10 & 7,30 & 3,32 & 2,16 & $0,04 *$ \\
\hline Zd & $-0,45$ & 3,51 & 0,08 & 4,05 & $-0,67$ & 0,50 \\
\hline Afr & 0,46 & 0,16 & 0,44 & 0,18 & 0,58 & 0,56 \\
\hline Populares & 3,95 & 1,82 & 3,83 & 1,72 & 0,29 & 0,77 \\
\hline $\mathrm{H}$ & 1,55 & 1,05 & 1,66 & 1,53 & $-0,46$ & 0,64 \\
\hline (H) & 1,20 & 1,47 & 0,63 & 0,99 & 1,73 & 0,10 \\
\hline Hd & 0,45 & 0,60 & 1,40 & 1,54 & $-7,02$ & $0,00 *$ \\
\hline (Hd) & 0,05 & 0,22 & 0,26 & 0,52 & $-4,20$ & $0,00 *$ \\
\hline A & 7,90 & 2,97 & 7,13 & 2,64 & 1,15 & 0,26 \\
\hline Art & 0,85 & 1,46 & 0,90 & 1,34 & $-0,15$ & 0,88 \\
\hline $\mathrm{Cg}$ & 1,25 & 1,33 & 0,53 & 1,07 & 2,41 & $0,02 *$ \\
\hline Idio & 1,10 & 1,02 & 0,60 & 0,85 & 2,19 & $0,04 *$ \\
\hline Sum6CCEE & 0,90 & 1,02 & 1,13 & 1,13 & $-1,00$ & 0,32 \\
\hline SumPond 6 & 3,15 & 3,91 & 2,73 & 3,27 & 0,48 & 0,63 \\
\hline $\mathrm{AG}$ & 0,15 & 0,37 & 0,13 & 0,34 & 0,24 & 0,81 \\
\hline $\mathrm{COP}$ & 0,50 & 0,76 & 0,56 & 0,89 & $-0,35$ & 0,72 \\
\hline PER & 0,90 & 0,39 & 0,53 & 1,30 & $-6,24$ & $0,00 *$ \\
\hline
\end{tabular}

\section{Discusión}

Los resultados obtenidos señalan que los indicadores Rorschach del grupo de delincuentes sexuales difieren significativamente de los datos del estudio de referencia nacional y de los datos de delincuentes comunes. Puesto que se trata de individuos que comparten una misma cultura y las condiciones de administración y puntuación de la prueba fueron las mismas para todos los evaluados, las diferen- cias encontradas pueden atribuirse a características psicológicas asociadas a la personalidad del delincuente sexual.

En el área interpersonal, los delincuentes sexuales presentan datos que dan cuenta de un estilo pasivo, superficial e infantil en sus contactos, presentando bajo interés hacia las personas y menor capacidad para involucrarse en relaciones interpersonales significativas. De esto se desprende que los delincuentes sexuales poseen menor comprensión 
y sensibilidad hacia quienes les rodean, mostrando dificultades para identificarse con otros, es decir, falta de empatía, lo que puede ser interpretado como un indicador de desadaptación social.

Estos datos evidencian la incompetencia del delincuente sexual en su funcionamiento social, tal como señalan Beneyto y Garrido (1997), lo cual le impide establecer relaciones satisfactorias con otros adultos. Algunos autores (Echeburúa \& Guerricaechevarría, 2000) señalan que esta falta de habilidad social en los delincuentes sexuales puede estar relacionado con el abuso sexual vivido en la infancia, lo que se asociaría al desarrollo de sentimientos de inferioridad e inhibiciones internas, con la consecuente incapacidad para establecer relaciones sociales normales.

Por otro lado, los delincuentes sexuales no aparecen defensivos y no tienden a sobre simplificar la información de la realidad (Lambda, F bajo). En el área emocional, el grupo de delincuentes sexuales aparece con una afectividad caracterizada por una menor tendencia a experimentar o reconocer en ellos estados de ansiedad (Y bajo) lo que, unido a la constricción ante la expresión de los afectos (FC y CF bajos), implicaría una defectuosa adaptación al medio. Tienden, entonces, a enfrentarse al entorno con reacciones pasivas y con una limitada vida afectiva (pasivos y SumPondC), lo que corresponde a una personalidad emocionalmente pobre, insensible a la estimulación de los demás.

En el área cognitivo-intelectual, el grupo de delincuentes sexuales, al ser comparado con los datos del estudio de referencia nacional, presenta un bajo ajuste perceptivo a la realidad $(\mathrm{X}+\%)$ que los hace más subjetivos en su forma de percibir los estímulos, ya sea que estos incluyan o no elementos afectivos. Esto implica que interpretan la realidad basándose en su propio mundo interno (infantil e inmaduro como anteriormente se describía), más que en los aspectos concretos u objetivos de esta, es decir, poseen una visión personal del mundo que interfiere con su capacidad de percibir realísticamente.

Se sugiere que la capacidad de adaptación al entorno en los delincuentes sexuales se caracteriza por ser deficiente, en el sentido que estos presentan inmadurez y un ajuste bajo a la realidad ( $\mathrm{X}+\%$ bajo), lo que interfiere con una adecuada interacción y apreciación con su entorno y de quienes lo rodean. Estos datos evidencian, por lo tanto, la necesidad de considerar en la elaboración de programas de tratamiento el desarrollo de habilidades sociales adecuadas y la confrontación de las distorsiones cognitivas de la realidad.

En esta línea de trabajo se consideraría que las distorsiones pueden traducirse en minimización o negación del delito o de su responsabilidad en este. Así, será necesario, como señalan Beneyto y Garrido (1997), instruir al delincuente sexual para reestructurar cognitivamente su interpretación de los fallos, enseñándole a pensar y evaluar críticamente su propio pensamiento, e incorporar en los tratamientos técnicas para enseñarles a pensar lógica, objetiva y racionalmente, sin sobre generalizar, distorsionar los hechos o externalizar la culpa.

Al comparar a los delincuentes sexuales con los delincuentes comunes, se observan también diferencias en el área interpersonal y el área cognitivointelectual. En relación al área interpersonal, los delincuentes sexuales serían menos retraídos y suspicaces con respecto al medio que los rodea (Hd menor), presentando una menor ansiedad social, al compararlos tanto con el grupo de referencia nacional como con los delincuentes comunes, situación que les predispondría a no tener una actitud de alerta y a ser menos cautelosos con respecto a sus actos y sus consecuencias.

Asimismo, los delincuentes sexuales presentan un tipo de vínculo en el que priman los contactos pasivos, dependientes (Cg alto) y autoritarios (PER). Además, aparece una menor probabilidad de modulación afectiva ( $\mathrm{FC}$ bajo).

Este estilo relacional inmaduro, carente de habilidades sociales adecuadas, da cuenta de la necesidad de dar especial atención en programas de tratamiento al análisis de situaciones interpersonales y de sus emociones, junto al desarrollo de habilidades sociales. Esto implicaría, como sostienen Beneyto y Garrido (1997), la valoración de situaciones de alto riesgo y habilidades de enfrentamiento, de manera que se incremente, entre otras habilidades, su capacidad de control.

En relación a los aspectos cognitivo-intelectuales, el grupo de delincuentes sexuales, comparado con los delincuentes comunes, presenta una alta motivación ante la tarea (Zf alto) pero una percepción marcada por la presencia de puntos de vista muy personales $(\mathrm{Xu} \%$ alto) y un tipo de acercamiento a la realidad que busca abarcarlo todo (W), lo cual implica mayor esfuerzo y no siempre mayor efectividad. Ello se traducirá en un ajuste perceptivo y un contacto inadecuado y poco práctico con su entorno. 
Entonces, la combinación alta motivación y baja productividad y falta de eficacia, puede llevarlos a frustrarse en el logro de sus metas y ¿a buscar compensar en otros ámbitos?

De este modo, la inadecuada capacidad observada en el grupo de delincuentes sexuales para establecer relaciones sociales aparece como una característica relevante en su funcionamiento, que se acentúa con su percepción fantaseada, pasividad y enfoque poco práctico de su entorno. La inadecuación relacional no aparece en los delincuentes comunes, lo que podría explicarse en función de que este grupo suele organizarse dentro de una estructura social particular, con jerarquía y roles claramente definidos. En estos últimos surgen los grupos o pandillas en torno a un objetivo común, como es el delinquir, mientras que los delincuentes sexuales comúnmente actúan solos, sin referentes que les permita contrastar la realidad, ni otro a quien puedan seguir o deban responderle.

De acuerdo con lo anterior, es posible señalar un conjunto de características de personalidad del grupo de delincuentes sexuales estudiados, que permite enunciar un perfil psicológico de los mismos, coincidente en algunos aspectos y diferente en otros con lo señalado teóricamente.

La muestra de delincuentes sexuales tiende a presentar una percepción distorsionada de la realidad, unido a un distanciamiento de lo convencional. Se observa en ellos un desarrollo cognitivo preponderantemente concreto y rígido, con baja capacidad de creatividad y simbolización. Se aprecia, además, dificultad para relacionarse de manera práctica, objetiva, activa y maduramente, tanto con elementos del entorno como con otras personas. Aparecen con una capacidad de control menor de lo que cabría esperar para la mayoría de los adultos, siendo posible, así, que no elaboren ni puedan llevar adecuadamente a la acción algunas de sus decisiones, con tendencia a reaccionar con frustración, inmadurez e impulsividad.

Estas características encontradas pueden entenderse como una confirmación de lo señalado por Barudy (1999), al describir al delincuente sexual como una persona inmadura, dependiente y con trastornos de las capacidades relacionales, con dificultades para establecer relaciones interpersonales e incomprensión de los valores que rigen la convivencia humana.

La pasividad, inmadurez y el bajo monto de recursos (EA) pueden entenderse al relacionarlos con las características del medio en que se desen- vuelven, señaladas por Cooper (1994), en cuanto a que un $98 \%$ de los delincuentes se asocia a la extrema pobreza y a la pobreza. Los delincuentes sexuales poseen una baja capacidad de control de sus impulsos, probablemente influidos por el hecho de vivir en un medio que no les posibilita un óptimo acceso a bienes, beneficios y posibilidades dentro de la sociedad de consumo. Lo anterior no les permitiría satisfacer adecuadamente sus necesidades y vivenciarían esto con una sensación de descontrol, que se acentúa por su falta de recursos. Es así que presentan dificultades para adaptarse a situaciones nuevas o inesperadas, ante las que no son capaces de reaccionar asertivamente; su repertorio de conductas pasivas, rutinarias y simples con las que comúnmente tienden a reaccionar no les resulta eficaz, descontrolándose entonces con facilidad en circunstancias con un mínimo de complejidad, con el riesgo de caer en formas impulsivas de pensamiento y acción. Por otro lado, factores como la falta de acceso al ámbito educacional o laboral se relacionan de manera importante con la falta de expectativas y proyectos a futuro y, por ende, con una mayor frustración, lo que los lleva a la búsqueda de satisfacciones inmediatas de sus necesidades crónicamente insatisfechas.

La falta de empatía (M bajo) puede entenderse en base a lo señalado por Friedlander en 1967, quien postuló que la falta de modelos y figuras significativas adecuadas -gratificadoras, cálidas, capaces de establecer límites claros- influye negativamente en la vida adulta del individuo. De esto se puede deducir que los delincuentes sexuales no aprendieron a vincularse ni a valorar la vida humana, ya que ellos no fueron a su vez apreciados y acogidos; así, el dañar una vida humana aparece como otra alternativa viable en la búsqueda de la satisfacción de sus necesidades y como una forma de validarse ante los demás. A la base de esto se encuentran también fuertes sentimientos de inseguridad, asociados, en parte, a la misma incapacidad para relacionarse de manera genuina y comprometida con los otros, lo que implica falta de empatía y consideración por el resto, tal como lo observó Endara (1960) en su estudio.

Al considerar el déficit en la capacidad de control, el bajo monto de recursos que no permiten satisfacer sus necesidades internas y la ausencia de una actitud francamente agresiva (bajo AG), se desprende que no existiría en el grupo de delincuentes estudiados la disposición interna o impulso definido a agredir sino que, por sus escasos recursos, son fácilmente vulnerables a desorganizarse ante situaciones de estrés y/o tensión, lo cual, unido a la incapacidad 
de posponer la satisfacción de sus necesidades y controlar sus impulsos, los lleva a buscar alternativas inmediatas de gratificación.

Sumado a lo anterior, destaca la ausencia tanto de una actitud abiertamente agresiva hacia los demás como de un marcado interés o desinhibición sexual, que pudiera explicar el comportamiento sexual alterado. Esto evidencia que no existiría una disposición francamente negativa o erotizada hacia el resto, como frecuentemente se señala en la literatura.

Por último, es posible señalar algunos elementos presumiblemente comunes a la delincuencia general, en base a las semejanzas más relevantes encontradas entre los grupos de delincuentes sexuales y delincuentes comunes estudiados. No aparecen motivados hacia las respuestas convencionales, otorgando así un menor valor a las normas sociales, tendiendo a ignorar o distorsionar la realidad cuando así conviene a sus necesidades. Además, su afectividad aparece restringida y empobrecida, por lo que demuestran bajo interés en establecer vínculos interpersonales profundos y significativos.

\section{Conclusiones}

A partir del análisis de resultados del test de Rorschach, es posible concluir la existencia de una tendencia en los delincuentes sexuales estudiados a presentar algunas características psicológicas comunes que permiten elaborar su perfil psicológico. Este perfil se compone esencialmente de siete rasgos característicos, los cuales son: 1) una baja capacidad de control de impulsos, 2) junto a una limitada cantidad de recursos que 3 ) no le permiten adaptarse adecuadamente a su entorno y 4) un estilo relacional pasivo e inmaduro. Además, poseen 5) una marcada tendencia a fantasear, que los lleva a mostrar un 6) enfoque poco práctico y una 7) percepción distorsionada de la realidad.

Cabe destacar que no se observa en los delincuentes sexuales estudiados una actitud abiertamente agresiva o de interés sexual hacia los demás, lo que revela que no existiría una preconcepción del medio como cargado de agresividad o erotización, que pudiera explicar su comportamiento abusivo, como cabría suponer, sino, más bien, estas respuestas surgen principalmente de la distorsión, falta e inadecuación en sus recursos e incompetencia social.

Considerando estas características, surgen algunos aspectos necesarios de considerar en la elaboración de programas de tratamiento. Entre estos destacan: el desarrollo de habilidades sociales, la confrontación de las distorsiones cognitivas de la realidad, el logro de una percepción madura y real de su entorno y de sí mismo y potenciación de recursos, principalmente afectivos.

Es necesario considerar el reducido tamaño de la muestra de este estudio, la que, por estar además compuesta exclusivamente por individuos pertenecientes a la Región de la Araucanía, no permite generalizar los resultados al resto de la población penal nacional de delincuentes sexuales. El estudio es entonces solo una primera y parcial aproximación al tema. Otra limitante del estudio hace referencia a que los resultados tampoco son generalizables a toda la población de agresores sexuales, ya que fueron obtenidos de una muestra de delincuentes detenidos, condenados y recluidos en un centro penitenciario.

Con base a la posible existencia de características comunes en los delincuentes sexuales que los distingue del resto de la población penal, se evidencia la necesidad de desarrollar criterios de intervención diferenciales dentro del sistema penitenciario que contribuyan a la evitación de la recaída del delincuente sexual, considerando fundamentalmente promover el análisis lógico de sus distorsiones cognitivas, fomentar el logro de competencias sociales y propiciar el desarrollo de una percepción madura y real de su entorno, potenciando así estrategias de control y reflexión previa a la acción.

\section{Referencias}

Barudy, J. (1999). Maltrato infantil. Ecología social: prevención y reparación. Santiago, Chile: Galdoc.

Beneyto, M. J. \& Garrido, V. J. (1997). La valoración psicológica de los agresores sexuales: los delitos, la reincidencia y el tratamiento. Cuadernos de Derecho Judicial: Delitos contra la Libertad Sexual, 7, 451-502.

Bouzat, P. \& Pinatel, J. (1975). Traité de droit pénal et de criminologie. Paris: Dalloz.

Bravo, L. (1982). La perspectiva clínica en la criminología contemporánea. Caracas: Editorial de Ciencias Jurídicas y Políticas.

Calderón, M., Duguet, W., Espinoza, C. \& Fuentealba, C. (2001). Características psicosociales de agresores sexuales que cursan condena entre 1999 y 2000 en cuatro centros de cumplimiento penitenciario de la IX Región. Tesis no publicada para optar al título de Psicólogo y al grado de Licenciado en Psicología, Universidad de La Frontera, Temuco, Chile.

Cooper, D. (1994). Delincuencia común en Chile. Santiago, Chile: LOM.

Echeburúa, E. (1994). Delitos sexuales: reacciones psicológicas en las víctimas de violación y tratamiento de los ofensores sexuales. Cuadernos de Derecho Judicial: Aportaciones de la Psicología al Ámbito Jurídico, 19, 209-226.

Echeburúa, E. \& Guerricaechevarría, C. (2000). Abuso sexual en la infancia: víctimas y agresores. Un enfoque clínico. Barcelona: Ariel. 
Endara, J. (1960). Psicodiagnóstico de Rorschach. Quito: Casa de la Cultura Ecuatoriana.

Espejo, M., Jiménez, P. \& Mella, C. (1996). Características psicológicas de una población penal homicida de sexo masculino observables a través del Test de Rorschach: un estudio exploratorio. Tesis no publicada para optar al título de Psicólogo y al grado de Licenciado en Psicología, Universidad de la Frontera, Temuco, Chile.

Exner, J. (1994). El Rorschach. Un sistema comprehensivo (Vol. 1). Madrid: Psimática.

Exner, J. \& Tuttle, K. (1994) Rorschach Interpretation Assistance Program Version 3. Lutz, FL: Psychological Assessment Resources.

Friedlander, K. (1967). Psicoanálisis de la delincuencia juvenil. Buenos Aires: Paidós.

Garrido, V. (1993). Técnicas de tratamiento para delincuentes. Madrid: Centro de Estudios Ramón Areces.

Garrido, V., Stangeland, P. \& Redondo, S. (1999). Principios de criminología. Valencia: Tirant Lo Blanch.

Gendarmería de Chile (2001). Principales delitos de la población reclusa condenada. Santiago, Chile: Autor. Extraído el 5 Septiembre, 2002, de http://www.gendarmeriadechile.cl

Gómez, M. (1999). La libertad condicional: peritación psicológica de los agresores sexuales. Papeles del Psicólogo, $73,41-50$.

Hermosilla, A. (1997). Variables sociodemográficas presentes en los internos condenados por delitos sexuales en los centros de cumplimiento penitenciario de la Araucanía. Un estudio exploratorio. Tesis no publicada para optar al título de Psicólogo y al grado de Licenciado en Psicología, Universidad de Temuco, Chile.

La Nación (2007, Agosto 9). El 62\% de los delitos sexuales son contra menores de edad. La Nación, País. Extraído el 16 Abril, 2009, de http://www.lanacion.cl/prontus_noticias/site/ edic/2007_08_09_1/home/home.html

Miotto, N. (1989, Julio). Características del psicodiagnóstico de Rorschach en homicidas conforme a los resultados de las investigaciones efectuadas en el trienio 1986-87-88. Ponencia presentada en el VII Congreso Latinoamericano de Rorschach y Otras Técnicas Proyectivas, Buenos Aires, Argentina.

Montoya, D., Díaz, R., Reyes, F., Abusleme, C. \& Garrido, J. (2004). Peritaje médico legal en delitos sexuales: una pauta práctica para su correcta realización. Revista Chilena de Obstetricia y Ginecología. 69, 55-59.

Romero, J. \& Luengo, M. A. (1999). Personalidad y delincuencia. Entre la biología y la sociedad. Granada: Grupo Editorial Universitario.

Sierra, J. C., Jiménez, E. \& Buela-Casal, G. (2006). Psicología forense: manual de técnicas y aplicaciones. Madrid: Biblioteca Nueva.

Sobral, J., Arce, R. \& Prieto, A. (1994). Manual de psicología jurídica. Barcelona: Paidós.
Vinet, E. (1995). El sistema comprehensivo del Rorschach: Un método sistemático de evaluación de la personalidad. Terapia Psicológica, 23, 35-51.

Vinet, E. (2000). El Sistema Comprehensivo del Rorschach en una muestra chilena de no-pacientes: estadísticos descriptivos y su interpretación psicológica. Terapia Psicológica, 7, 143-157.

Weigle, C. (1988). Como interpretar el Rorschach. Su articulación con el psicoanálisis. Buenos Aires: Artigas Suárez.

Wolfgang, M. \& Ferracutti, F. (1971). La subcultura de la violencia: hacia una teoría criminológica. Ciudad de México: Fondo de Cultura Económica.

\section{Anexo}

Delitos sexuales, según la Ley $N^{o} 19.617$

Violación: acceso carnal, vaginal, anal o bucal, mediando fuerza, intimidación, privación de sentido o imposibilidad de resistir.

Estupro: acceso carnal, vaginal, anal o bucal de una persona entre 12 y 18 años en que se abuse de trastorno mental, relación de dependencia, grave desamparo o engaño, abusando de su inexperiencia sexual.

Abuso sexual: realizar cualquier acto de significación sexual y de relevancia, con contacto corporal o, a lo menos, afectación genital, anal o bucal de la víctima, aun cuando no hubiere contacto corporal con ella: (a) con una persona, sea hombre o mujer, por medio de la fuerza o aprovechándose de la imposibilidad de la víctima de resistir el acto; (b) con un menor entre 12 y 18 años, aprovechándose de circunstancias, prevalimiento (abuso de autoridad) o engaño; (c) con un menor de 12 años, aunque no exista contacto corporal.

Sodomía: relaciones homosexuales con un menor de edad.

Violación conyugal: la ley considera que una persona, aunque esté casada, conserva su libertad sexual y es libre de aceptar o rechazar la posibilidad de tener relaciones sexuales con su cónyuge.

Fecha de recepción: Marzo de 2008.

Fecha de aceptación: Marzo de 2009. 